\title{
KETOGENIC DIET TREATMENT FOR STATUS EPILEPTICUS
}

\author{
CARMEN SANDU ${ }^{1,2 *}$, SANDA ADRIANA MAGUREANU ${ }^{1}$, CATRINEL ILIESCU ${ }^{1,2}$, CRISTINA \\ POMERAN $^{1,2}$, DANA CRISTINA CRAIU ${ }^{1,2}$
}

${ }^{1}$ Pediatric Neurology II Discipline, Clinical Neurosciences Department, "Carol Davila” University of Medicine and Pharmacy, Bucharest, Romania

${ }^{2}$ Pediatric Neurology Clinic, "Prof. Dr. Alexandru Obregia" Clinical Hospital, 10, Berceni Avenue, $4^{\text {th }}$ District, 041914,

Bucharest, Romania

*corresponding author: carmensandu_u6@yahoo.com

Manuscript received: November 2018

\begin{abstract}
The ketogenic diet (KD) is a high fat normoproteic, low carbohydrate diet considered in recent years as an alternative treatment option for patients with refractory status epilepticus (RSE) or super-refractory status epilepticus (SRSE), both, in children and adults. The aim of current research is to review and compare the efficacy of KD in children and adults with RSE and SRSE regardless of underlying aetiology. We performed a retrospective PubMed search between June 1997 until $30^{\text {th }}$ of October 2017 and selected all articles in which KD was used for the treatment of children and adults with status epilepticus refractory to standard therapy. 120 patients (adults and children) with RSE and SRSE were identified. An important proportion of patients $(92 / 120 \sim 77 \%, 64$ children and 28 adults) responded to KD therapy. In conclusion, KD is proving to be a valuable therapeutic option in the intensive care units for the treatment of RSE and SRSE in children and adults, available data showing efficacy mostly but not limited to (febrile infection related to encephalopathy in school aged children) FIRES in children and encephalitis in adults. Specific protocols and training should be made available in intensive care units in order to implement and manage $\mathrm{KD}$ in children and adults with status epilepticus who do not respond to standard therapeutic approaches.
\end{abstract}

\section{Rezumat}

Dieta cetogenă (DC) este o dietă bogată în grăsimi, normoproteică şi cu un conținut sărac de carbohidrați, considerată în ultimii ani drept o opțiune de tratament pentru pacienții cu status epilepticus refractar (RSE) sau super-refractar (SRSE), atât la copii, cât și la adulți. Scopul cercetării actuale este de a analiza datele existente în literatură şi de a compara eficienţa DC la copii și adulți cu RSE şi SRSE, indiferent de etiologie. S-a efectuat o cercetare retrospectivă a bazei de date PubMed din iunie 1997 pînă la 30 octombrie 2017, fiind selectate toate articolele în care DC a fost utilizată pentru tratamentul copiilor şi adulților cu status epilepticus refractar la terapia standard. Au fost identificați 120 de pacienți (adulți și copii) cu RSE şi SRSE. O proporție importantă de pacienți (92/120 77\%, 64 copii și 28 adulți) au răspuns la terapia cu DC. În concluzie, DC se dovedește a fi o opțiune terapeutică valoroasă în unitățile de terapie intensivă pentru tratamentul statusului epileptic refractar şi superrefractar la copii şi adulți, datele existente demonstrând eficienţa mai ales, dar nu exclusiv, în FIRES (febrile infection related encephalopathy in school-age children) la copii și encefalită la adulți. Protocoale specifice și instruire adecvată ar trebui să fie puse la dispoziție în unitățile de terapie intensivă pentru a implementa și administra DC la copiii şi adulții cu status epilepticus care nu răspund la abordările terapeutice standard.

Keywords: ketogenic diet, refractory status epilepticus, FIRES, encephalitis

\section{Introduction}

The ketogenic diet $(\mathrm{KD})$ is a high fat, normoproteic, low carbohydrate diet used for many years, in various protocols, for the treatment of drug-resistant epilepsy [40]. A high number of publications prove its efficacy, both in children [25, 28], and adults [27], when standard therapy is inefficient.

A guide for implementing and managing the KD in children was published in 2008 by an international group of KD experts, clinicians and dieticians, offering guidance for the main indications of the diet. Among these, specific epileptic syndromes, such as epileptic spasms, Dravet syndrome, tuberous sclerosis complex, Rett or Doose syndrome have particular benefit from the use of ketogenic diet. $\mathrm{KD}$ is the only treatment for special genetic syndromes such as Glut 1 deficiency syndrome or pyruvate dehydrogenase (PDH) deficiency syndrome $[18,13]$.

Meanwhile, cases of (refractory or super refractory) status epilepticus with favourable response to the ketogenic diet have also been reported [41].

According to International League Against Epilepsy (ILAE) - 1981, status epilepticus (SE) has been conceptually defined as a seizure "sufficiently prolonged or repeated at sufficiently brief intervals so as to produce an unvarying and enduring epileptic condition" [12]. 
The new definition of status epilepticus proposed by ILAE Task Force combines the operational and, respectively, the physiopathological definitions of SE as: "a condition resulting either from the failure of mechanisms responsible for seizure termination or from the initiation of mechanisms, which lead to abnormally prolonged seizures (aftertime point t 1 ) and can have long-term consequences(after time point t2), including neuronal death, neuronal injury, and alteration of neuronal networks, depending on the type of seizures" [36].

Refractory status epilepticus (RSE) is defined as the status continuing for more than 2 hours, despite benzodiazepines and other anticonvulsant medication (first- and second-line medication) and requiring general anaesthesia.

Super refractory status epilepticus (SRSE) is the last stage of the condition when the status continues or recurs for more than 24 hours, despite general anaesthesia [30]. Both conditions carry high rates of morbidity and mortality, compelling additional interventions. Ketogenic diet (KD) is considered as a "needed adjunct strategy for the management of status epilepticus" [29].

\section{Materials and Methods}

\section{Search criteria}

A systematic search of electronic medical database PubMed from June 1997 to the end of October 2017 has been performed using the following key words „ketogenic diet, status epilepticus” „ketogenic diet, FIRES" (febrile infection related encephalopathy in school-age children). The reference list with retrieved articles has been studied. Available abstracts or full texts, have been searched for clinical cases using ketogenic diet as treatment for status epilepticus. Eligibility criteria

We included articles presenting clinical cases of status epilepticus regardless of age or aetiology but using ketogenic diet in the therapeutic management that were all reported in the literature by $31^{\text {st }}$ of October 2017. All study types, retrospective and prospective, in English, French and Spanish have been considered. Review articles have also been included.

\section{Results and Discussion}

\section{Analysed data}

The search for „ketogenic diet and status epilepticus” returned 122 articles. An additional search using the keywords „ketogenic diet and FIRES” resulted in other 57 articles. Out of 29 articles in total, 10 referred to adults, 17 to children and 2 for both, adults and children. They have been analysed and 120 patients have been identified as using ketogenic diet in the management of status epilepticus. Only one article had a prospective design with a standardized protocol including 15 adults with super refractory status epilepticus [8]. Adults and children were analysed as independent categories and compared.

Adults

36 adults have been identified in 12 articles, listed in chronological order (Table I). In adults, the age varied between 18 - 82 years, with 2 patients of 18 years old reported by Villeneuve in 2009 and Cervenka in 2017 [8, 39]. The average age of the 35 patients was 40.3 years, one patient did not have the age mentioned ("middle-aged") [4]. Out of the total number of adult patients 22 were females and 14 males.

There were 2 types of status epilepticus reported, generalised and focal motor, with 2 exception, one case of myoclonic status epilepticus and one case of nonconvulsive status epilepticus (NCSE) [4, 21]. Regarding the underlying aetiology, 5 out of 36 cases $(\sim 14 \%)$ were encephalitis of unknown aetiology or NORSE (new onset refractory status epilepticus), 4 out of $36(11 \%)$ anti NMDA-R encephalitis or anoxic ischemic aetiology, 3 out of $36(\sim 8 \%)$ were intracranial haemorrhage, 2 out of $36(\sim 6 \%)$ viral encephalitis. There was also one case of each of the following: focal cortical dysplasia, glioblastoma multiforma, Rassmussen encephalitis, Ito syndrome or mitochondrial aetiology. The majority of cases (35 patients) used the classical KD, most of them $(33 / 35, \sim 94 \%)$ with a ratio of $4: 1$, in only one patient was administered the low glycaemic index diet (LGIT) [21]. The interval between the onset of status epilepticus and initiation of ketogenic diet varied between 2 and 101 days, with an average of 23 days for 34 patients. Positive results were achieved in 31 patients, with 28 of 31 ( $90 \%)$ patients achieving status remission and 3 patients $(\sim 10 \%)$ a decrease of seizure frequency (improvement). Side effects have been reported in 13 of $26(\sim 50 \%)$ patients, most of them developing various complications like metabolic acidosis (5/14), constipation (2/14), increased lipid profile (3/14), and hyponatremia (1/14). Ketosis has been achieved in 32 patients.

\section{Children}

84 children were identified from 19 articles (Table II). Their age varied between 0 and 16 years, with a median age of 7.83 years in 82 patients; in 2 children the age was not mentioned [22]. The youngest patient in which KD was initiated was 9 weeks old [11]. The gender was clearly stated in 82 patients, with 43 males $(\sim 52 \%)$ and 39 females ( 48\%).

9 cases with myoclonic status epilepticus $[7,5,10]$ and 3 cases with NCSE (nonconvulsive status epilepticus) have been described [19, 31]. FIRES (febrile-illness related epilepsy syndrome), was the 
FARMACIA, 2019, Vol. 67, 2

most common aetiology with 28/84 ( $33 \%)$ paediatric patients reported, 1 of them with associated PCDH19 and GABRG2 mutation [2]. There are also 4 reported patients with viral encephalitis, 3 patients with anti NMDA-R encephalitis, 5 patients with unknown aetiology and also isolated cases of variable aetiologies: structural, autoimmune, infectious, genetic or metabolic (Table

Table I

Adults

\begin{tabular}{|c|c|c|c|c|c|c|c|c|c|}
\hline $\begin{array}{c}\text { Author, year } \\
\text { (reference } \\
\text { number) }\end{array}$ & $\begin{array}{c}\text { Number } \\
\text { of } \\
\text { subjects }\end{array}$ & $\begin{array}{l}\text { Age } \\
\text { range } \\
\text { (years)/ } \\
\text { gender }\end{array}$ & Aetiology/diagnosis & $\begin{array}{c}\text { Type } \\
\text { of KD, } \\
\text { ratio }\end{array}$ & Ketosis & $\begin{array}{c}\text { Day of } \\
\text { initiation }\end{array}$ & $\begin{array}{c}\text { Time to } \\
\text { suppression/ } \\
\text { improvement } \\
\text { (days) }\end{array}$ & $\begin{array}{r}\text { Clinical } \\
\text { outcome }\end{array}$ & Side effects \\
\hline $\begin{array}{c}\text { Bodenant, } \\
2008[3] \\
\end{array}$ & 1 & $54, \mathrm{M}$ & $\begin{array}{c}\text { Pharmacorezistant } \\
\text { epilepsy }\end{array}$ & $\begin{array}{c}\mathrm{KD} \\
(4: 1) \\
\end{array}$ & uk & 31 & 7 & $\begin{array}{c}\text { SE remission } \\
(1 / 1) \\
\end{array}$ & uk \\
\hline $\begin{array}{c}\text { Villeneuve, } \\
2009 \text { [39] }\end{array}$ & 1 & $18, \mathrm{M}$ & $\begin{array}{l}\text { Hypomelanosis of } \\
\text { ITO syndrome }\end{array}$ & $\begin{array}{c}\mathrm{KD} \\
(4: 1)\end{array}$ & yes & uk & 3 & $\begin{array}{c}\text { SE remission } \\
(1 / 1)\end{array}$ & uk \\
\hline $\begin{array}{l}\text { Wusthoff, } \\
2010 \text { [41] }\end{array}$ & 2 & $\begin{array}{l}29 \mathrm{~F} \\
34 \mathrm{M}\end{array}$ & $\begin{array}{c}\text { Rasmussen, Viral } \\
\text { encephalitis }\end{array}$ & $\begin{array}{l}\mathrm{KD} \\
(4: 1)\end{array}$ & yes & $\begin{array}{l}18- \\
101\end{array}$ & $8-11$ & $\begin{array}{c}\text { SE remission } \\
(2 / 2)\end{array}$ & uk \\
\hline $\begin{array}{l}\text { Cervenka, } \\
2011[9]\end{array}$ & 1 & $54, \mathrm{M}$ & $\begin{array}{c}\text { Idiopathic } \\
\text { (possibly } \\
\text { autoimmune) }\end{array}$ & $\begin{array}{l}\text { KD } \\
(4: 1)\end{array}$ & yes & 58 & uk & $\begin{array}{c}\text { SE remission } \\
(1 / 1)\end{array}$ & uk \\
\hline $\begin{array}{c}\text { Martikainen, } \\
2011[21]\end{array}$ & 1 & $26, \mathrm{~F}$ & $\begin{array}{c}\text { Mitochondrial } \\
\text { (POLG1) }\end{array}$ & LGIT & uk & 7 & 4 & $\begin{array}{c}\text { SE remission } \\
(1 / 1)\end{array}$ & uk \\
\hline $\begin{array}{c}\text { Nam, } \\
2011[24]\end{array}$ & 1 & $40, \mathrm{~F}$ & Viral encephalitis & $\begin{array}{c}\mathrm{KD} \\
(4: 1)\end{array}$ & yes & 15 & 30 & $\begin{array}{c}\text { SE remission } \\
(1 / 1)\end{array}$ & none \\
\hline $\begin{array}{c}\text { Strzelczyk, } \\
2013 \text { [33] }\end{array}$ & 1 & $21, \mathrm{~F}$ & Lafora disease & $\begin{array}{c}\text { KD } \\
\text { iv } \\
(4: 1)\end{array}$ & yes & 16 & uk & improvement & uk \\
\hline $\begin{array}{l}\text { Thakur, } \\
2014 \text { [35] }\end{array}$ & 10 & $\begin{array}{l}23- \\
51 \\
6 \mathrm{~F} \\
4 \mathrm{M}\end{array}$ & $\begin{array}{c}4 \text { Encephalitis of } \\
\text { unknown aetiology, } \\
2 \text { NMDA-R } \\
\text { encephalitis, LGI1 } \\
\text { encephalitis, } \\
\text { Cortical dysplasia, } \\
\text { Neurocysticercosis, } \\
\text { Anoxic ischemic } \\
\text { aetiology }\end{array}$ & $\begin{array}{l}\text { KD } \\
(4: 1- \\
9 / 10 \\
3: 1)\end{array}$ & $\begin{array}{l}\text { yes } \\
9 / 10\end{array}$ & $\begin{array}{c}2-60 \\
\text { median } \\
21.5\end{array}$ & $\begin{array}{c}\text { 1-31, } \\
\text { median } 3\end{array}$ & $\begin{array}{l}\text { SE remission } \\
(9 / 10)\end{array}$ & $\begin{array}{l}2 \mathrm{HTG} \\
1 \text { acidosis }\end{array}$ \\
\hline $\begin{array}{c}\text { Cash, } 2015 \\
{[4]}\end{array}$ & 1 & $\begin{array}{l}\text { middle } \\
\text { age, } \mathrm{M}\end{array}$ & $\begin{array}{l}\text { Hypoxic ischemic } \\
\text { brain injury }\end{array}$ & $\begin{array}{c}\mathrm{KD} \\
(4: 1)\end{array}$ & yes & 36 & uk & improvement & uk \\
\hline $\begin{array}{c}\text { Amer, } 2015 \\
{[1]}\end{array}$ & 1 & $21, \mathrm{~F}$ & $\begin{array}{c}\text { NMDA-R } \\
\text { encephalitis }\end{array}$ & $\begin{array}{c}\text { KD } \\
(4: 1)\end{array}$ & yes & 21 & na & improvement & uk \\
\hline $\begin{array}{c}\text { Uchida, } 2017 \\
\text { [37] }\end{array}$ & 1 & $20, \mathrm{~F}$ & $\begin{array}{c}\text { NMDA-R } \\
\text { encephalitis }\end{array}$ & $\mathrm{KD}$ & uk & uk & 60 & $\begin{array}{c}\text { SE remission } \\
(1 / 1)\end{array}$ & uk \\
\hline $\begin{array}{l}\text { Cervenka, } \\
2017[8]^{*}\end{array}$ & 15 & $\begin{array}{l}18- \\
82 \\
5 \mathrm{M}, \\
10 \mathrm{~F}\end{array}$ & $\begin{array}{c}5 \text { NORSE, } 2 \\
\text { Anoxic ischemic } \\
\text { injury, } 2 \text { LGS, } 3 \\
\text { Intracranial } \\
\text { haemorrhage, } \\
\text { Encephalitis of } \\
\text { unknown aetiology, } \\
\text { Epilepsy secondary } \\
\text { to SBS, GBM }\end{array}$ & $\begin{array}{l}\mathrm{KD} \\
(4: 1)\end{array}$ & yes & $\begin{array}{c}2-39 \\
\text { median } \\
10\end{array}$ & $\begin{array}{c}0-10 \\
\text { median } 5\end{array}$ & $\begin{array}{c}\text { SE remission } \\
(11 / 15)\end{array}$ & $\begin{array}{c}3 \text { metabolic } \\
\text { acidosis } \\
2 \text { hypoglycaemia } \\
1 \text { hyponatremia } \\
1 \text { weight loss } \\
1 \text { constipation } \\
1 \text { HLD } \\
1 \text { metabolic } \\
\text { acidosis and } \\
\text { hypoglycaemia } \\
1 \text { constipation } \\
\text { and HLD } \\
\end{array}$ \\
\hline
\end{tabular}

Abbreviations: $\mathrm{KD}=$ ketogenic diet; $\mathrm{M}=$ male; $\mathrm{F}=$ female; $\mathrm{uk}=$ unknown; na = not applicable; NMDA-R = N-methyl-D-aspartate receptor; NORSE $=$ new-onset refractory status epilepticus of unknown aetiology; POLG $1=$ mitochondrial polymerase $\mathrm{c}$ related epilepsy; LGI1 $=$ leucine-rich, glioma-inactivated 1 encephalitis; LGS = Lennox Gastaut Syndrome; GBM = glioblastoma multiforma; HTG = high triglycerides; HLD = hyperlipidaemia; $\mathrm{SBS}=$ shaken-baby syndrome.

Nearly all patients, $82 / 84(\sim 98 \%)$ followed the classical KD with variable ratios $(1: 1$ to $6: 1)$, mostly $4: 1,75 / 82(\sim 91 \%)$ patients. 2 patients have followed the Modified Atkins Diet (MAD) [19]. KD was initiated after an average of 32 days from onset of SE for 58/84 ( $69 \%)$ patients.
This ranged from day 1 to day $420[2,24]$. The clinical response, defined as suppression of status epilepticus or decrease of frequency and/or duration of seizure was positive in $72 / 84(\sim 86 \%)$ patients, with $45 / 72(\sim 63 \%)$ patients achieving suppression of status epilepticus, 8/72 ( 11\%) between $75-99 \%$ seizure improvement, $11 / 72 \quad(15 \%) \quad 50-74 \%$ 
FARMACIA, 2019, Vol. 67, 2

seizure improvement and 8/72 ( 11\%) patients under $50 \%$ seizure improvement or shorter seizure duration. Data about side effects related to $\mathrm{KD}$ has been reported in 48 patients, with 22 patients reporting adverse effects, mostly constipation 5/21, high triglycerides 4/21, vomiting 4/21, gastroesophageal reflux (GER) 2/21, hypoglycaemia $4 / 21$, pancreatitis $3 / 21$ or weight loss $1 / 21$. Data about ketosis has been retrieved in 77 patients, $75 / 77$ achieving ketosis after KD initiation.

Table II

Children

\begin{tabular}{|c|c|c|c|c|c|c|c|c|c|}
\hline $\begin{array}{l}\text { Author, year } \\
\text { (reference } \\
\text { number) }\end{array}$ & $\begin{array}{c}\text { Number } \\
\text { of } \\
\text { subjects }\end{array}$ & \begin{tabular}{|c|} 
Age \\
range \\
(years)/ \\
gender \\
\end{tabular} & Aetiology/diagnosis & $\begin{array}{c}\text { Type of } \\
\text { KD, } \\
\text { ratio }\end{array}$ & Ketosis & $\begin{array}{c}\text { Day of } \\
\text { initiation }\end{array}$ & \begin{tabular}{|c|} 
Time to \\
suppression/ \\
improvement \\
(days) \\
\end{tabular} & $\begin{array}{l}\text { Clinical } \\
\text { outcome }\end{array}$ & Side effects \\
\hline $\begin{array}{l}\text { Villeneuve, } \\
2009 \text { [39] }\end{array}$ & 4 & $\begin{array}{l}1-10 \\
1 \mathrm{M}, 3 \mathrm{~F}\end{array}$ & \begin{tabular}{|c|}
2 Cryptogenic, Sturge- \\
Weber syndrome, Grey \\
matter encephalitis
\end{tabular} & $\begin{array}{l}\mathrm{KD} \\
(4: 1)\end{array}$ & uk & uk & $\begin{array}{c}1-10, \text { median } \\
3.75\end{array}$ & $\begin{array}{c}\text { SE remission } \\
(3 / 4)\end{array}$ & uk \\
\hline $\begin{array}{c}\text { Kumada, } 2010 \\
{[19]}\end{array}$ & 2 & $4-5, F$ & $\begin{array}{c}\text { Frontal lobe epilepsy, } \\
\text { Subcortical band } \\
\text { heterotopia }\end{array}$ & MAD & yes & uk & $\begin{array}{c}5-10 \\
\text { median } 7.5\end{array}$ & $\begin{array}{c}\text { SE remission } \\
(2 / 2)\end{array}$ & uk \\
\hline $\begin{array}{c}\text { Nabbout, } 2010 \\
{[23]} \\
\end{array}$ & 9 & $\begin{array}{c}5-8 \\
5 \mathrm{~F}, 4 \mathrm{M}\end{array}$ & FIRES & $\begin{array}{c}\mathrm{KD} \\
(4: 1) \\
\end{array}$ & yes & \begin{tabular}{|c|}
$4-55$ \\
median 23 \\
\end{tabular} & $\begin{array}{c}4-6, \text { median } \\
4.8 \\
\end{array}$ & \begin{tabular}{|c|}
$\begin{array}{c}\text { SE remission } \\
(7 / 9)\end{array}$ \\
\end{tabular} & uk \\
\hline $\begin{array}{c}\text { Ismail, } 2011 \\
{[16]} \\
\end{array}$ & 1 & $14, \mathrm{~F}$ & FIRES & $\begin{array}{c}\mathrm{KD} \\
(4: 1)\end{array}$ & uk & 120 & 10 & \begin{tabular}{|c|} 
SE remission \\
$(1 / 1)$
\end{tabular} & uk \\
\hline Nam, 2011 [24] & 4 & $\begin{array}{c}4-14 \\
2 \mathrm{~F}, 2 \mathrm{M}\end{array}$ & Viral encephalitis & $\begin{array}{l}\mathrm{KD} \\
(4: 1)\end{array}$ & yes & $\begin{array}{c}30-420 \\
\text { median } \\
150\end{array}$ & $\begin{array}{c}3-19 \\
\text { median } 11\end{array}$ & \begin{tabular}{|l|} 
SE remission \\
$(1 / 4)$ \\
$90 \% \mathrm{sz}$ \\
reduction \\
$(1 / 4)$ \\
$75 \% \mathrm{sz}$ \\
reduction \\
$(2 / 4)$
\end{tabular} & $\begin{array}{c}1 \text { Aspiration } \\
\text { pneumonia and } \\
\text { constipation } \\
1 \text { Constipation } \\
\text { and severe GER } \\
1 \mathrm{HTG}, \\
\text { constipation, } \\
\text { GER } \\
1 \text { Constipation }\end{array}$ \\
\hline $\begin{array}{c}\text { Vaccarezza, } \\
2012[38]\end{array}$ & 5 & $\begin{array}{c}1-14 \\
1 \mathrm{~F}, 4 \mathrm{M}\end{array}$ & \begin{tabular}{|c|}
3 FIRES, \\
Hemimegaloencephaly, \\
Chiari syndrome type 2 \\
\end{tabular} & $\begin{array}{l}\text { KD } \\
(4: 1)\end{array}$ & yes & $\begin{array}{c}15-52 \\
\text { median } 32\end{array}$ & $\begin{array}{c}1-3 \\
\text { median } 2\end{array}$ & $\begin{array}{c}\text { SE remission } \\
(4 / 4)\end{array}$ & uk \\
\hline Sort, 2013 [32] & 3 & $\begin{array}{l}3-11 \\
2 \mathrm{M}, 1 \mathrm{~F}\end{array}$ & $\begin{array}{l}\text { HHES, Mitochondrial } \\
\text { respiratory chain } \\
\text { enzyme defect, FIRES }\end{array}$ & $\begin{array}{l}\text { KD } \\
(4: 1)\end{array}$ & yes & $\begin{array}{l}6-48 \\
\text { median } \\
\mathrm{A}^{*}\end{array}$ & $\begin{array}{c}1-13 \\
\text { median } 7\end{array}$ & \begin{tabular}{|c|} 
SE remission \\
$(2 / 3)$ \\
No response \\
$(1 / 3)$ \\
\end{tabular} & $\begin{array}{c}1 \text { Weight loss, } \\
\text { HTG }\end{array}$ \\
\hline $\begin{array}{c}\text { Caraballo, } 2014 \\
{[6]}\end{array}$ & 10 & $\begin{array}{l}0-16 \\
6 \mathrm{M}, 4 \mathrm{~F}\end{array}$ & $\begin{array}{c}\text { NMDA-R encephalitis, } \\
\text { Hemimegaloencephaly, } \\
2 \text { FCD, } \\
4 \text { Refractory focal } \\
\text { seizures associated with } \\
\text { febrile illness, } 2 \\
\text { Unknown }\end{array}$ & $\begin{array}{l}\mathrm{KD} \\
(4: 1)\end{array}$ & yes & uk & median 5 & $\begin{array}{c}75-90 \% \mathrm{sz} \\
\text { reduction } \\
(2 / 10) \\
50-75 \% \mathrm{sz} \\
\text { reduction } \\
(5 / 10) \\
<50 \% \\
\text { szreduction } \\
(3 / 10) \\
\end{array}$ & $\begin{array}{l}1 \text { Vomiting and } \\
\text { hypoglycaemia } \\
2 \text { Pancreatitis }\end{array}$ \\
\hline $\begin{array}{c}\text { O'Connor, } 2014 \\
{[26]}\end{array}$ & 5 & $\begin{array}{c}0-10 \\
3 \mathrm{M}, 2 \mathrm{~F}\end{array}$ & $\begin{array}{l}\text { Alpers Hutenlocher } \\
\text { syndrome, } \\
\text { Mitochondrial defect } \\
\text { and influenza A, } 2 \\
\text { Symptomatic } \\
\text { generalized epilepsy, } \\
\text { Unknown } \\
\end{array}$ & $\begin{array}{l}\text { KD } \\
(4: 1)\end{array}$ & yes & $\begin{array}{l}4-22 \\
\text { median } \\
12.8\end{array}$ & $\begin{array}{c}2-8 \\
\text { median } 4.6\end{array}$ & $\begin{array}{c}\text { SE remission } \\
(5 / 5)\end{array}$ & none \\
\hline $\begin{array}{c}\text { Singh, } 2014 \\
{[31]}\end{array}$ & 2 & $\begin{array}{l}7-10 \\
1 \mathrm{M}, 1 \mathrm{~F}\end{array}$ & FIRES & $\begin{array}{c}\mathrm{KD} \\
(4: 1 \\
6: 1) \\
\end{array}$ & yes & $\begin{array}{c}3-13 \\
\text { median } 8\end{array}$ & $\begin{array}{l}2-20 \\
\text { median } 10\end{array}$ & $\begin{array}{c}\text { SE remission } \\
(2 / 2)\end{array}$ & uk \\
\hline $\begin{array}{c}\text { Caraballo, } 2015 \\
{[7]}\end{array}$ & 2 & $1-2, \mathrm{M}$ & $\begin{array}{c}\text { Progressive } \\
\text { encephalopathy, } \\
\text { Unknown }\end{array}$ & $\begin{array}{l}\mathrm{KD} \\
(4: 1)\end{array}$ & yes & 30 , uk & 7 & $\begin{array}{c}75-90 \% \mathrm{sz} \\
\text { reduction } \\
(1 / 2) \\
50 \% \mathrm{sz} \\
\text { reduction } \\
(1 / 2)\end{array}$ & none \\
\hline $\begin{array}{c}\text { Cobo, } 2015 \\
{[11]}\end{array}$ & 4 & $\begin{array}{l}0-13 \\
1 \mathrm{~F}, 3 \mathrm{M}\end{array}$ & $\begin{array}{c}\text { MMPSI, TSC, } 2 \\
\text { Cryptogenic }\end{array}$ & $\begin{array}{l}\mathrm{KD} \\
(2: 1, \\
3.2: 1, \\
4: 1- \\
2 / 4)\end{array}$ & yes & $\left|\begin{array}{c}19-67 \\
\text { median } 35\end{array}\right|$ & $\begin{array}{c}2-8 \\
\text { median } 5.3\end{array}$ & \begin{tabular}{|c|}
$50 \% \mathrm{sz}$ \\
reduction \\
$(1 / 3)$ \\
Shorter \\
seizures $(2 / 4)$ \\
No \\
improvement \\
$(1 / 4)$
\end{tabular} & $\begin{array}{c}1 \text { Nephrolithiasis } \\
1 \text { Asymptomatic } \\
\text { hypoglycaemia } \\
1 \text { Constipation, } \\
\text { GER }\end{array}$ \\
\hline
\end{tabular}


FARMACIA, 2019, Vol. 67, 2

\begin{tabular}{|c|c|c|c|c|c|c|c|c|c|}
\hline $\begin{array}{c}\text { Author, year } \\
\text { (reference } \\
\text { number) }\end{array}$ & $\begin{array}{c}\begin{array}{c}\text { Number } \\
\text { of } \\
\text { subjects }\end{array} \\
\text { subje }\end{array}$ & $\begin{array}{c}\text { Age } \\
\text { range } \\
\text { (years)/ } \\
\text { gender } \\
\end{array}$ & Aetiology/diagnosis & $\begin{array}{c}\text { Type of } \\
\text { KD, } \\
\text { ratio }\end{array}$ & Ketosis & $\begin{array}{c}\text { Day of } \\
\text { initiation }\end{array}$ & $\begin{array}{c}\text { Time to } \\
\text { suppression/ } \\
\text { improvement } \\
\text { (days) }\end{array}$ & $\begin{array}{l}\text { Clinical } \\
\text { outcome }\end{array}$ & Side effects \\
\hline Fung, 2015 [15] & 4 & $\begin{array}{l}6-16 \\
2 \mathrm{M}, 2 \mathrm{~F}\end{array}$ & $\begin{array}{c}\text { NORSE, VGKC } \\
\text { encephalitis, FIRES, } \\
\text { Encephalitis of possible } \\
\text { autoimmune origin }\end{array}$ & $\begin{array}{l}\mathrm{KD} \\
(4: 1)\end{array}$ & $\begin{array}{l}\text { yes } \\
3 / 4\end{array}$ & $\begin{array}{l}12-18 \\
\text { median } \\
17.5\end{array}$ & 5 & $\begin{array}{c}\text { SE remission } \\
\quad(1 / 4) \\
\text { No response } \\
\quad(3 / 4)\end{array}$ & \begin{tabular}{|c|}
1 Falling \\
plasma proteins \\
1 Vomiting \\
1 increase \\
seizure
\end{tabular} \\
\hline Lin, 2015 [20] & 1 & $6, \mathrm{M}$ & NORSE & $\begin{array}{l}\mathrm{KD} \\
(4: 1) \\
\end{array}$ & yes & 1.5 & 1 & $\begin{array}{c}\text { SE remission } \\
(1 / 1)\end{array}$ & uk \\
\hline $\begin{array}{c}\text { Millichap, } 2015 \\
{[22]}\end{array}$ & 2 & uk & FIRES & $\begin{array}{c}\mathrm{KD} \\
(4: 1)\end{array}$ & uk & uk & uk & $\begin{array}{c}\text { SE remission } \\
(2 / 2)\end{array}$ & uk \\
\hline $\begin{array}{c}\text { Appavu, } 2016 \\
{[2]}\end{array}$ & 10 & $\begin{array}{l}2-16 \\
4 \mathrm{~F}, 6 \mathrm{M}\end{array}$ & \begin{tabular}{|c|} 
Rasmussen encephalitis \\
Generalized epilepsy, \\
intractable \\
LGS \\
Non-ketotic \\
hyperglycinaemia \\
Mycoplasma post- \\
infectious \\
encephalitis \\
NORSE \\
FIRES \\
FIRES and PCDH19, \\
GABRG2 mutation \\
2 NMDA-R encephalitis \\
\end{tabular} & $\begin{array}{c}\mathrm{KD}(4: 1 \\
-9 / 10 \\
5: 1)\end{array}$ & $\begin{array}{l}\text { yes } \\
9 / 10\end{array}$ & $\left|\begin{array}{c}1-45 \\
\text { median } 20\end{array}\right|$ & $\begin{array}{c}1-19 \\
\text { median } 7\end{array}$ & $\begin{array}{c}\text { SE remission } \\
\quad(9 / 10) \\
\begin{array}{c}\text { No response } \\
(1 / 10)\end{array}\end{array}$ & uk \\
\hline $\begin{array}{c}\text { Chiusolo, } 2016 \\
{[10]}\end{array}$ & 1 & $8, \mathrm{M}$ & Unknown & $\begin{array}{c}\mathrm{KD} \\
(3: 1)\end{array}$ & yes & uk & na & $\begin{array}{c}\text { No response } \\
(1 / 1)\end{array}$ & $\begin{array}{c}1 \text { high liver } \\
\text { enzymes }\end{array}$ \\
\hline $\begin{array}{c}\text { Caraballo, } 2017 \\
{[5]}\end{array}$ & 6 & $\begin{array}{l}2,5-9 \\
4 \mathrm{~F}, 2 \mathrm{M}\end{array}$ & $\begin{array}{l}\text { CDKL5, SCN8A, } 2 \\
\text { Angelman, Rett, } \\
\text { Unknown }\end{array}$ & $\begin{array}{l}\mathrm{KD} \\
(4: 1)\end{array}$ & yes & uk & uk & $\begin{array}{c}75-99 \% \mathrm{sz} \\
\text { reduction } \\
(2 / 6) \\
50-74 \% \mathrm{sz} \\
\text { reduction } \\
(3 / 6) \\
<50 \% \mathrm{sz} \\
\text { reduction } \\
(1 / 6)\end{array}$ & 2 Vomiting \\
\hline $\begin{array}{c}\text { Farias-Moeller, } \\
2017[14]\end{array}$ & 9 & $\begin{array}{c}2-8 \\
6 \mathrm{~F}, 3 \mathrm{M}\end{array}$ & $\begin{array}{c}5 \text { Encephalitis/FIRES, } \\
2 \text { FIRES, Epileptic } \\
\text { encephalopathy, CNS- } \\
\text { HLF }\end{array}$ & \begin{tabular}{|c|}
$\mathrm{KD}$ \\
$(1: 1$ \\
$3: 1,4: 1$ \\
$-7 / 9)$
\end{tabular} & yes & $\left|\begin{array}{c}10-16 \\
\text { median } 13\end{array}\right|$ & 7 & $\begin{array}{c}\text { SE remission } \\
(5 / 9) \\
50 \% \mathrm{sz} \\
\text { reduction } \\
(1 / 9) \\
10 \% \mathrm{sz} \\
\text { reduction } \\
(2 / 9) \\
\text { Unknown } \\
(1 / 9)\end{array}$ & $\begin{array}{c}1 \mathrm{HTG} \\
1 \mathrm{HTG} \text { and } \\
\text { pancreatitis } \\
\text { *VPA }\end{array}$ \\
\hline
\end{tabular}

Abbreviations: $\mathrm{KD}=$ ketogenic diet; $\mathrm{MAD}=$ modified Atkins diet, $\mathrm{M}=$ male; $\mathrm{F}=$ female; $\mathrm{uk}=$ unknown; na $=$ not applicable; $\mathrm{sz}=\mathrm{seizure}$; FIRES = febrile infection related encephalopathy in school-age children, HHES = heiconvulsion hemiplegia epilepsy syndrome, NMDA-R $=\mathrm{N}$-methyl-D-aspartate receptor; FCD $=$ frontal cortical dysplasia, MMPSI $=$ malignant migrating partial seizures of infancy, TSC $=$ tuberous sclerosis complex, NORSE = new-onset refractory status epilepticus of unknown aetiology, VGKC = voltage gated potassium channel, LGS = Lennox Gastaut syndrome, PCDH $19=$ Protocadherin 19, GABRG2 = gamma-aminobutyric acid type A receptor gamma 2, CDKL5 = cyclin-dependent kinase-like 5, SCN8A = Sodium channel, voltage gated, type VIII, alpha subunit , CNS-HLF= central nervous system- hemophagocytic lymphohistiocytosis, GER = gastroesophageal reflux, HTG = high triglycerides, HLD = hyperlipidaemia, *VPA $=$ on valproic acid; $A^{*}$ KD tried twice (day 8, day 83).

In the last few years, KD gained more and more interest as a therapeutic option for emergency situations like refractory or super refractory status epilepticus. In a retrospective review of 10 publications from 2013, the authors reported a total of 32 children and adults receiving dietary treatment for status epilepticus, of which $78 \%$ (25/32) of patients achieved complete control of SE [17]. During our investigation, we found comparable results, with $73 / 120(\sim 61 \%)$ patients reported to achieve complete control of SE and 92/120 ( 77\%) more than $50 \%$ improvement, $17 / 120$ ( 23\%) patients have shown no improvement at all.

Regarding FIRES and febrile illness related epilepsy, Nabout and Kossoff considered that these entities should to be added to the list of indications in the next KD Expert Consensus Statement and that was recently updated with the new recommendations of the International Ketogenic Diet Study Group published in 2018 [13, 17]. FIRES was the most common aetiology found in children also, with $28 / 84$ patients reported, 21 of 
them $(\sim 75 \%)$ achieving complete remission of status epilepticus.

Apart from the beneficial role in the acute management of FIRES, there are authors outlining the importance of KD for the cognitive outcome, for the short, as well as for the long term one $[16,22$, 31, 41].

Although comparing results of using of $\mathrm{KD}$ in adults versus children is difficult due to the heterogeneous cohorts, the variable ways of initiation and associated treatments, it can be concluded that in adults, encephalitis, mostly autoimmune and viral, is the most reported entity $(14 / 34) \sim 39 \%$, with high rates of response, $12 / 14$ with complete status remission. The average interval from the onset of SE until the initiation of KD was 23 days in adults and 32 days in children, and still with good results. There are studies suggesting the initiation of $\mathrm{KD}$, earlier in the management of SE [2]. Refractory myoclonic SE is also considered an entity in which the KD should be commenced earlier in the course of the treatment. Cognitive deterioration may be avoided if seizures are well controlled [7].

In a recent prospective study of 15 adult patients treated with KD, a high number of patients, 11/14 patients $(79 \%)$ completing the therapy, achieved remission of status epilepticus [8].

Compliance in patients with status epilepticus is not an issue, due to their critical condition. The majority of children but adult patients too, followed classical KD, ratio 4:1, administered via gastrostomy or nasogastric tube. Some authors consider that children with seizures may benefit from IV (parenteral) KD due to enteral feeding intolerance [10].

A metanalysis of $\mathrm{Ye}$ and collaborators in 2015 showed that in adults with intractable epilepsy classical KD may be more effective but adult patients are less compliant with it than with a modified Atkins diet [42]. In our cohort, one adult patient followed LGIT with good seizure outcome [21] and 2 children followed MAD with similar results [19].

External factors, like „concurrent therapies” may contribute to remission of status epilepticus. The authors recognise the need for prospective trials, difficult to achieve for those patients in critical condition [2].

Specific recommendation (guidelines) with clear key points regarding when to consider starting KD for status epilepticus are made available by different authors [13, 18, 26, 34].

\section{Conclusions}

Our current research shows that KD can work in refractory and super-refractory status epilepticus. More than $60 \%$ of monitored patients responded by suppression of status epilepticus. Other retrospective studies with series of cases using KD as a treatment for SE and SRSE have been published, in adults [34], children [10] or both [17, 32]. At the time of publication there were few retrospective studies about using KD as a treatment method for SE and SRSE in adults, children or both but this is, by our knowledge, the largest study to gather children and adults. Specific protocols and training should be made available in intensive care units in order to implement and manage $\mathrm{KD}$ in children and adults with status epilepticus and no response to standard approaches.

\section{References}

1. Amer S, Shah P, Kommineni V, Refractory status epilepticus from NMDA receptor encephalitis successfully treated with an adjunctive ketogenic diet. Annals of Indian Academy of Neurology, 2015; 18: 256-257.

2. Appavu B, Vanatta L, Condie J, Kerrigan JF, Jarrar $\mathrm{R}$, Ketogenic diet treatment for pediatric superrefractory status epilepticus. Seizure, 2016; 41: 62-65.

3. Bodenant M, Moreau C, Sejourné C, Delval A, Cuisset JM, Derambure P, Destée A, Defebvre L, Interest of the ketogenic diet in a refractory status epilepticus in adults. Revue Neurologique, 2008; 164(2): 194-199.

4. Cash C, Use of the ketogenic diet in an adult with myoclonic status epilepticus. Clinical Nutrition, abstract ESPEN 10, 2015; e199-200.

5. Caraballo R, Darra F, Reyes G, Armeno M, Cresta A, Mestre G, Bernardina BD, The ketogenic diet in patients with myoclonic status in non-progressive encephalopathy. Seizure, 2017; 51: 1-5.

6. Caraballo RH, Flesler S, Armeno M, Fortini S, Agustinho A, Mestre G, Cresta A, Buompadre MC, Escobal N, Ketogenic diet in pediatric patients with refractory focal status epilepticus. Epilepsy Research, 2014; 108(10): 1912-1916.

7. Caraballo RH, Valenzuela GR, Armeno M, Fortini S, Mestre G, Cresta A, The ketogenic diet in two paediatric patients with refractory myoclonic status epilepticus. Epileptic Disorders, 2015; 17(4): 491-495.

8. Cervenka MC, Hocker SE, Koenig M, Bar B, Henry-Barron B, Kossoff EH, Hartman AL, Probasco JC, Benavides DR, Venkatesan A, Hagen EC, Dittrich D, Stern T, Radzik B, Depew M, Caserta FM, Nyquist P, Kaplan PW, Geocadin RG, A phase I/II multicenter ketogenic diet study for adult super-refractory status epilepticus. Neurology, 2017; 88: 938-943.

9. Cervenka MC, Hartman AL, Venkatesan A, Geocadin RG, Kossoff EH, The ketogenic diet for medically and surgically refractory status epilepticus in the neurocritical care unit. Neurocritical Care, 2011; 15(3): 519-524.

10. Chiusolo F, Diamanti A, Bianchi R, Fusco L, Elia M, Capriati T, Vigevano F, Picardo S, From intravenous to enteral ketogenic diet in PICU: A potential treatment strategy for refractory status 
FARMACIA, 2019, Vol. 67, 2

epilepticus. European Journal of Paediatric Neurology, 2016; 20(6): 843-847.

11. Cobo NH, Sankar R, Murata KK, Sewak SL, Kezele MA, Matsumoto JH, The ketogenic diet as broad-spectrum treatment for super-refractory pediatric status epilepticus: challenges in implementation in the pediatric and neonatal intensive care units. Journal of Child Neurology, 2015; 30: 259-266.

12. Commission on Classification and Terminology of the International League Against Epilepsy. Proposal for revised clinical and electroencephalographic classification of epileptic seizures. Epilepsia, 1981; 22: 489-501.

13. Eric Kossoff H, Zupec-Kania BA, Auvin S, BallabanGil KR, Christina Bergqvist AG, Blackford R, Buchhalter JR, Caraballo RH, Cross JH, Dahlin MG, Donner EJ, Guzel O, Jehle RS, Klepper J, Kang HC, Lambrechts DA, Liu YMC, Nathan JK, Nordli DR Jr, Pfeifer HH, Rho JM, Scheffer IE, Sharma S, Stafstrom CE, Thiele EA, Turner Z, Vaccarezza MM, van der Louw EJTM, Veggiotti P, Wheless JW, Wirrell EC; Charlie Foundation; Matthew's Friends; Practice Committee of the Child Neurology Society, Optimal clinical management of children receiving dietary therapies for epilepsy: Updated recommendations of the International Ketogenic Diet Study Group. Epilepsia Open, 2018; 3(2): 175-192.

14. Farias-Moeller R, Bartolini L, Pasupuleti A, Brittany Cines RD, Kao A, Carpenter JL, A practical approach to ketogenic diet in the pediatric intensive care unit for super-refractory status epilepticus. Neurocritical Care, 2017; 26(2): 267272.

15. Fung EL, Chang SK, Yam KK, Yau PY, Ketogenic diet as a therapeutic option in super-refractory status epilepticus. Pediatric Neonatology, 2015; 56(6): 429-431.

16. Ismail FY, Kossof EH, AERRPS, DESC, NORSE, FIRES: multilabeling or distinct epileptic entities? Epilepsia, 2011; 52(11): e185-e189.

17. Kossoff EH, Nabbout R, Use of dietary therapy for status epilepticus. Journal of Child Neurology, 2013; 28: 1049-1051.

18. Kossoff EH, Zupec-Kania BA, Amark PE, Ballaban-Gil KR, Christina Bergqvist AG, Blackford R, Buchhalter JR, Caraballo RH, Helen Cross J, Dahlin MG, Donner EJ, Klepper J, Jehle RS, Kim HD, Christiana Liu YM, Nation J, Nordli DR Jr, Pfeifer HH, Rho JM, Stafstrom CE, Thiele EA, Turner Z, Wirrell EC, Wheless JW, Veggiotti $\mathrm{P}$, Vining EP; Charlie Foundation, Practice Committee of the Child Neurology Society; Practice Committee of the Child Neurology Society; International Ketogenic Diet Study Group, Optimal clinical management of children receiving the ketogenic diet: recommendations of the International Ketogenic Diet Study Group. Epilepsia, 2009; 50: 304-317.

19. Kumada T, Miyajima T, Kimura N, Saito K, Shimomura H, Oda N, Fujii T, Modified Atkins diet for the treatment of nonconvulsive status epilepticus in children. Journal of Child Neurology, 2010; 25(4): 485-489.

20. Lin JJ, Lin KL, Chan OW, Hsia SH, Wang HS, CHEESE Study Group, Intravenous ketogenic diet therapy of acute stage of super-refractory status epilepticus in a pediatric patient. Pediatric Neurology, 2015; 52(4): 442-445.

21. Martikainen $M H$, Päivärinta $M$, Jääskeläinen $S$, Majamaa K, Successful treatment of POLG-related mitochondrial epilepsy with antiepileptic drugs and low glycaemic index diet. Epilepsia, 2011; 52: e181-e184.

22. Millichap JJ, Millichap JG, Ketogenic diet as preferred treatment of FIRES. Pediatric Neurololy Briefs, 2015; 29(1): 3.

23. Nabbout R, Mazzuca M, Hubert P, Peudennier S, Allaire C, Flurin V, Aberastury M, Silva W, Dulac $\mathrm{O}$, Efficacy of ketogenic diet in severe refractory status epilepticus initiating fever induced refractory epileptic encephalopathy in school age children (FIRES). Epilepsia, 2010; 51: 2033-2037.

24. Nam SH, Lee BL, Lee CG, Yu HJ, Joo EY, Lee J, Lee $\mathrm{M}$, The role of ketogenic diet in the treatment of refractory status epilepticus. Epilepsia, 2011; 52: e181-e184.

25. Neal EG, Chaffe H, Schwartz RH, Lawson MS, Edwards N, Fitzsimmons G, Whitney A, Cross JH, The ketogenic diet for the treatment of childhood epilepsy: a randomised controlled trial. Lancet Neurol., 2008; 7: 500-506.

26. O'Connor SE, Ream MA, Richardson C, Mikati MA, Trescher WH, Byler DL, Sather JD, Michael EH, Urbanik KB, Richards JL, Davis R, Zupanc ML, Zupec-Kania B, The ketogenic diet for the treatment of pediatric status epilepticus. Pediatric Neurology, 2014; 50: 101-103.

27. Payne NE, Cross JH, Sander JW, Sisodiya SM, The ketogenic and related diets in adolescents and adults - A review. Epilepsia, 2011; 52: 1941-1948.

28. Sharma S, Sankhyan N, Gulati S, Agarwala A, Use of the modified Atkins diet for treatment of refractory childhood epilepsy: a randomized controlled trial. Epilepsia, 2013; 54: 481-486.

29. Shorvon S, Ferlisi M, The outcome of therapies in refractory and super-refractory convulsive status epilepticus and recommendations for therapy. Brain, 2012; 135: 2314-2328.

30. Shorvon S, Ferlisi M, The treatment of superrefractory status epilepticus: a critical review of available therapies and a clinical treatment protocol. Brain, 2011; 134: 2802-2818.

31. Singh RK, Joshi SM, Potter DM, Leber SM, Carlson MD, Shellhaas RA, Cognitive outcomes in febrile infection-related epilepsy syndrome treated with the ketogenic diet. Pediatrics, 2014; 134(5): e1431-e145.

32. Sort R, Born A, Pedersen KN, Fonsmark L, Uldall $\mathrm{P}$, Ketogenic diet in 3 cases of childhood refractory status epilepticus. European Journal of Paediatric Neurology, 2013; 17: 531-536.

33. Strzelczyk A, Reif PS, Bauer S, Belke M, Oertel WH, Knake S, Rosenow F, Intravenous initiation and maintenance of ketogenic diet: proof of 
concept in super-refractory status epilepticus. Seizure, 2013; 22(7): 581-583.

34. Tanya Williams J, Cervenka MC, The role for ketogenic diets in epilepsy and status epilepticus in adults. Clinical Neurophysiology Practice, 2017; 2: 154-160.

35. Thakur KT, Probasco JC, Hocker SE, Roehl K, Henry B, Kossoff EH, Kaplan PW, Geocadin RG, Hartman AL, Venkatesan A, Cervenka MC, Ketogenic diet for adults in super-refractory status epilepticus. Neurology, 2014; 82: 665-670.

36. Trinka E, Cock H, Hesdorffer D, Rossetti AO, Scheffer IE, Shinnar S, Shorvon S, Lowenstein DH, A definition and classification of status epilepticus-Report of the ILAE Task Force on Classification of Status Epilepticus. Epilepsia, 2015; 56:1515-23.

37. Uchida Y, Kato D, Toyoda T, Oomura M, Ueki Y, Ohkita K, Matsukawa N, Combination of ketogenic diet and stiripentol for super-refractory status epilepticus: A case report. Journal of the Neurological Sciences, 2017; 373: 35-37.

38. Vaccarezza M, Silva W, Maxit C, Agosta G, Superrefractory status epilepticus: treatment with ketogenic diet in pediatrics. Revista de Neurologia, 2012; 55(1): 20-25.

39. Villeneuve N, Pinton F, Bahi-Buisson N, Dulac O, Chiron C, Nabbout R, The ketogenic diet improves recently worsened focal epilepsy. Developmental Medicine \& Child Neurology, 2009; 51(4): 276281.

40. Wilder RM, The effects of ketonemia on the course of epilepsy. Mayo Clin Bull, 1921; 2: 307-308.

41. Wusthoff CJ, Kranick SM, Morley JF, Bergqvist AGC, The ketogenic diet in treatment of two adults with prolonged nonconvulsive status epilepticus. Epilepsia, 2010; 51:1083-1085.

42. Ye F, Li XJ, Jiang WL, Sun HB, Liu J, Efficacy of and patient compliance with a ketogenic diet in adults with intractable epilepsy: a meta-analysis. Journal of Clinical Neurology, 2015; 11(1): 26-31. 